\title{
La psicología educativa colombiana: un tránsito hacia el desarrollo
}

\author{
Colombian educational psychology: \\ a path towards development
}

Karen Ángel Gómez*

Recibido: 17 de abril de 2021 / Aceptado: 4 de junio de 2021 / Publicado: 13 de diciembre de 2021

Forma de citar este artículo en APA:

Ángel Gómez, K. (2021). La psicología educativa colombiana: un tránsito hacia el desarrollo. Poiésis, (41), 143-148. https://doi.org/10.21501/16920945.4182

\section{Resumen}

La psicología educativa ha sido objeto de diversos cambios a lo largo del tiempo y, al llegar a la Universidad Nacional de Colombia en 1948, los rasgos teóricos y paradigmáticos locales reflejaban, en alguna medida, lo que a nivel macro estaba ocurriendo dentro de la disciplina. Este rastreo teórico acerca del proceso de tránsito, crítica y cambio de la psicología educativa da un parámetro para entender de dónde viene, dónde está y hacia dónde va a nivel nacional. Como conclusión, se evidencia un tránsito hecho por la psicología educativa colombiana hacia un desarrollo, fortalecimiento, establecimiento y posicionamiento en un contexto cada vez más cambiante y exigente a través de los diversos paradigmas, metodologías, técnicas y modelos educativos existentes y desarrollados en la historia de la educación.

\section{Palabras clave:}

Colombia; Desarrollo de la educación; Historia; Psicología de la educación; Paradigma; Psicología educativa; Prospección educacional; Tendencia educacional. 


\section{Abstract}

Educational psychology has undergone various changes over time and, upon arriving at the National University of Colombia in 1948, the local theoretical and paradigmatic features reflected, to some extent, what was happening at the macro level within the discipline. This theoretical tracking on the process of transition, criticism and change of educational psychology gives a parameter to understand where it comes from, where it is and where it is heading at national level. In conclusion, there is evidence of a transition made by Colombian educational psychology towards a development, strengthening, establishment and positioning in an increasingly changing and demanding context through the various paradigms, methodologies, techniques and educational models existing and developed in the history of education.

\section{Keywords:}

Colombia; Development of education; History; Educational psychology; Paradigm; Educational psychology; Educational prospecting; Educational trend. 
La psicología educativa ha sido objeto de diversos cambios a lo largo del tiempo. Al llegar a la Universidad Nacional de Colombia en 1948 los rasgos teóricos y paradigmáticos locales reflejaban, en alguna medida, lo que a nivel macro estaba ocurriendo dentro de la disciplina. Este rastreo teórico acerca del proceso de tránsito, crítica y cambio de la psicología educativa da al lector un parámetro para entender de dónde viene, dónde está y hacia dónde va a nivel nacional.

El inicio de la psicología educativa, se da gracias a la unión, a través del tiempo, de los estudios en educación y los estudios en filosofía, medicina y psicología, que tenían como objetivo el poder mejorar las "pedagogías, las didácticas, los currículos, y a entender de forma inter y transdiciplinariamente la educación" (Erazo Santander, 2012, p. 143). Para esto, desde los años treinta, la educación en el ámbito colombiano hacía énfasis en los paradigmas científicos con los estudios en psicometría para selección, evaluación de inteligencia o aptitud y orientación vocacional; esta última gracias a la influencia que la psicología empezaba a tener sobre su práctica (Bernal de Sierra, 1999, p. 105). Este paradigma nacional reflejaba lo que autores extranjeros como Thorndike, Skinner, Pavlov, Bruner y Dewey hacían desde la psicología experimental y científica para el fortalecimiento de los métodos de enseñanza con el paradigma instruccional centrado en el profesor, la instrucción programada para alumnos o el asociacionista-conductista (Beltrán Llera \& Pérez Sánchez, 2011, p. 208).

Seguidamente, las próximas décadas trajeron para la disciplina cambios importantes no solo para su constitución sino también para sus planteamientos y paradigmas. Con respecto a esto, en la década de los cincuenta se fundó en la Universidad Nacional de Colombia el primer centro de psicología y comenzaron a surgir los jardines infantiles creados por los mismos psicólogos de la universidad (Bernal de Sierra, 1999, p. 107) como forma de mantener activo el ciclo de formación educativo con el ingreso constante de estudiantes de todos los grados; acto reflejado en autores como el pedagogo alemán Federico Froebel, creador de estos centros en su país nativo desde el siglo XVIII y XIX (Ramos Valdez, 2007, p. 61). Ya en la década del sesenta y setenta, se reconocería en Colombia la psicología educativa como una disciplina oficial y necesaria para la educación desde una perspectiva científica y experimental, en la que las escuelas normales, estatales y privadas no solo estaban centradas en el rendimiento académico sino también en el estudio del desarrollo mental (Bernal de Sierra, 1999, p. 106). A pesar de esto, desde Beltrán Llera y Pérez Sánchez (2011), en las últimas décadas mencionadas, la psicología educativa afuera del país cruzaba por cambios paradigmáticos, temáticos y metodológicos importantes, como la crítica de los modelos pedagógicos más conductistas, instruccionales, lineales y rígidos con su posterior reemplazo por paradigmas más cognitivos, abiertos y flexibles. Por esto, comenzando los años noventa, surgió en la disciplina una crisis de identidad/status y una necesidad de ampliación paradigmática y metodológica por la ineficacia en su rol y por la disminución de su influencia en la educación (p. 207). Sin embargo, gracias a las continuas críticas, se retomaron autores como Thorndike, Piaget, Ausubel, Brown y Palincsar, Pressley y Levin, Sternberg, Gardner, Goleman y Dweck, que fortalecieron la educación en el ámbito de los métodos y explicaciones del aprendizaje para poder entender cómo aprendían los estudiantes, cómo transformaban la información aprendida y cómo la relacionaban con las experiencias pasadas a través del paradigma personal, el conexionismo, el aprendizaje cognitivo y metacognitivo, la enseñanza centrada en el alumno y su procesamiento de 
la información en la construcción de significados, el reconocimiento del profesor como mediador y evaluador de procesos y el estudio de la motivación, la inteligencia emocional, las inteligencias múltiples y las creencias personales, donde su objetivo era "ayudar a aprender" (pp. 211-219). Además, se evidencia que el tomar en cuenta el contexto de lo que se enseña y se aprende fue influenciado por la psicología ecológica y la psicología ambiental, donde se considera importante el reconocimiento del ambiente educativo y la interpretación de la conducta del alumno desde este mismo, en el que, desafortunadamente, se encuentran varias "barreras del aprendizaje, barreras que el contexto establece y que dificultan el acceso de muchos estudiantes al aprendizaje" (López Escribano \& Fernández Sánchez, 2014, p. 8).

Lo anterior nos lleva a señalar el reflejo de lo que ocurría en el ámbito nacional, donde, como consecuencia de los rápidos cambios paradigmáticos y estructurales de la disciplina, las intervenciones de la psicología educativa colombiana comienzan también a ser ineficientes al seguir utilizando paradigmas ahistóricos, limitados y descontextualizados que no lograban responder a las necesidades de la comunidad educativa, como por ejemplo de los estudiantes, que han enfrentado desde siempre diferentes problemáticas personales, psicológicas y sociales como el fracaso escolar, problemas orgánicos, cognitivos, emocionales, de personalidad, neuropsicológicos, de la conducta, sexuales, familiares o de drogadicción (Erazo Santander, 2012, p. 141); y a ser confusas al no tener claro la "contribución que los psicólogos escolares ofrecen en el apoyo a niños, colegios y familias" (Farrell, 2009, p. 74). Precisamente, para ampliar su campo de acción, la psicología educativa se dirigió de un modelo exclusivamente médico a uno sistémico, en el que se enfatiza en la resolución colaborativa de los problemas educativos desde una perspectiva ecológica, cultural, multidisciplinar y flexible para atender y hacer consulta ya no solo a los niños, sino también a todos los estudiantes, sus familias y trabajar en conjunto con los profesionales de la misma escuela (Farrell, 2009). A pesar de esto, Uribe Aramburo (2013), psicólogo y docente de la Facultad de Psicología y Ciencias Sociales de la Universidad Católica Luis Amigó, señaló que, en la práctica, se podía encontrar una gran confusión o desconocimiento de parte de los mismos psicólogos educativos sobre su rol y sobre las variadas posibilidades de su campo de acción, ya que estos parecen repetir las mismas funciones tradicionales una y otra vez, haciendo que la psicología educativa sea irrelevante e ineficaz en su actuar en el contexto actual. Para esto, recuerda Uribe Aramburo, que hay tres áreas de actuación del psicólogo educativo, las cuales son (1) la evaluación y el psicodiagnóstico con actividades psicométricas y roles más tradicionales, (2) la psicopedagogía y la psicohigiene con actividades y roles que cumplen una función de staff o asesoría más enfocada en los procesos de la institución educativa como la "evaluación y rediseño de los contenidos curriculares y metodologías de enseñanza" (p. 141), y (3) la psico orientación que tiene actividades y roles dirigidos a la orientación individual de estudiantes, docentes y familias y una promoción y prevención de distintas problemáticas psicosociales. Con respecto a esto y gracias a los cambios y clarificaciones, la educación colombiana superior fue incorporando el Bienestar Universitario y los programas de inducción para estudiantes nuevos hechos por psicólogos educativos en conjunto con la comunidad escolar. 
En la actualidad, Colombia y otros países como México, Argentina, Perú, Chile, España y Estados Unidos han profesionalizado la psicología educativa, abriendo líneas de investigación, estudios especializados, fomentando una base teórica más robusta, desde una perspectiva interdisciplinaria e implementando roles más inclusivos desde un enfoque psicosocial, de género, subjetivo, investigativo e inclusivo, reflejado en la definición de educación de la Ley 115, 1994, art. 9, en la cual se plantea un proceso de formación permanente e integral del ser humano, teniendo en cuenta el contexto y los sistemas que lo rodean y recalcando la importancia de su dignidad, de sus derechos y de sus deberes (p. 1). Se evidencia que la psicología educativa ha avanzado, cambiado y se ha transformado mucho en el transcurso de los años, tomando en cuenta las críticas hechas a su modelo más reduccionista y centrado en pruebas, para convertirse en lo que es actualmente, una disciplina que aborda la vida escolar con una mirada más global (Bernal de Sierra, 1999, p. 110). Por tanto, y en consonancia con Beltrán Llera y Pérez Sánchez (2011),

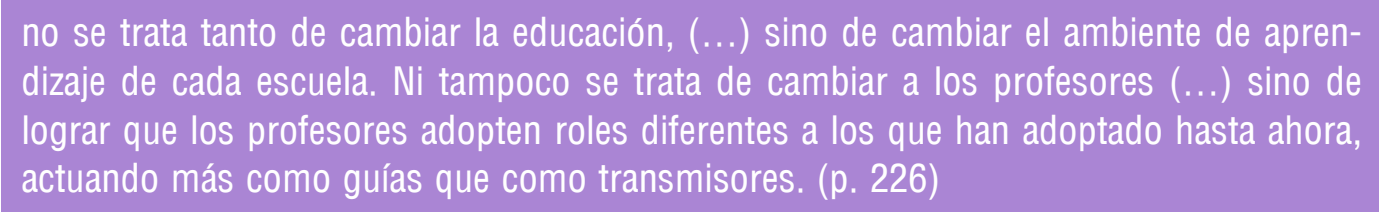

En conclusión, se evidencia, desde el rastreo teórico, un tránsito hecho por la psicología educativa colombiana -reflejando lo que ocurría afuera del país- hacia un desarrollo, fortalecimiento, establecimiento y posicionamiento de la disciplina, ampliando sus horizontes paradigmáticos desde el paradigma instruccional hasta el personal, metodológicos desde el método pedagógico del conductismo hasta las diferentes perspectivas del cognitivismo, y técnicos desde los instrumentos y dinámicas de enseñanza cerrados, lineales y rígidos hasta los más abiertos, flexibles, comprensivos y con un claro objetivo hacia el desarrollo y no solo al abastecimiento del conocimiento de un área en específico por parte del alumno.

Se recomienda al lector hacer un proceso investigativo con énfasis en esta dicotomía educativa, su influencia en la educación actual y la que podría tener a futuro, teniendo en cuenta que no es necesario llegar al eclecticismo para encontrar puntos medios y fortalecer, teórica y prácticamente, la disciplina.

\section{Conflicto de intereses}

La autora declara la inexistencia de conflicto de interés con institución o asociación comercial de cualquier índole. 


\section{Referencias}

Beltrán Llera, J. A., \& Pérez Sánchez, L. (2011). Más de un siglo de psicología educativa. Valoración general y perspectivas de futuro. Papeles del Psicólogo, 32(3), 204-231. http://www. papelesdelpsicologo.es/pdf/1981.pdf

Bernal de Sierra, F. Á. (1999). La psicología escolar: cincuenta años [Edición especial]. Revista Colombiana de Psicología, 105-111. https://revistas.unal.edu.co/index.php/psicologia/article/ view/32148

Congreso de Colombia. (8 de febrero de 1994). Ley General de Educación. [Ley 115 de 1994]. D0: 41.214 .

Erazo Santander, 0. A. (2012). Reflexiones sobre la psicología educativa. Revista de Psicología GEPU, 3(2), 139-157.

Farrell, P. (2009). El papel en desarrollo de los psicólogos escolares y educativos en el apoyo a niños, escuelas y familias. Papeles del Psicólogo, 30(1), 74-85. http://www.papelesdelpsicologo.es/ pdf/1658.pdf

López Escribano, C., \& Fernández Sánchez, C. (2015). Jesús A. Beltrán Llera (1936-2014). Bordón. Revista de Pedagogía, 67(3), 7-12. https://recyt.fecyt.es/index.php/BORDON/article/ view/36615

Ramos Valdez, A. (2007). Algunos protagonistas de la pedagogía; vida y obra de grandes maestros. Universidad Pedagógica Nacional.

Uribe Aramburo, N. (2013). Fundamentos teóricos y funciones del psicólogo educativo. Revista Colombiana de Ciencias Sociales, 4(1), 135-149. https://www.funlam.edu.co/revistas/index. php/RCCS/article/view/955/877 